\title{
Wide-Area Current Differential Protection Based on DSP
}

\author{
Lan Yang ${ }^{1, \mathrm{a}}$, Can Bao ${ }^{1}$, Jinbo Lan ${ }^{2}$, Jianfei Yang ${ }^{1}$ and Huaren $\mathrm{Wu}^{1}$ \\ ${ }^{1}$ School of Electrical and Automation Engineering,Nanjing Normal University,210042 Nanjing, China \\ ${ }^{2}$ Guodian Nanjing Automation co., LTD, Nanjing 211100, China \\ 1,ayanglanszt@163.com
}

Keywords: WAP, Current differential Protection, DSP, RT-LAB.

Abstract. The paper introduces the development status of wide-area protection (WAP) and the widearea current differential protection (WACDP) algorithm. Digital signal processor (DSP) TMS320F28335 is used as the controller, the system model is built with MATLAB/Simulink, combined with semi physical simulation experiment platform RT-LAB, and based on the graph theory to realize wide area current differential protection.

\section{Introduction}

With the development of the power systems,the composition of power grids becomes increasingly complex, putting forward higher requirements of reliability and stability of the power systems . Relay protection is the first line of defense to protect the safety of power grids and an important guarantee of the safe and stable operation of power systems. Action from the protection device must be correct, fast and reliable in order to minimize the affect when fault occurs. However, performance of conventional relay is limited by offline setting when balancing between the sensitivity and selectivity. Moreover, protecting only a single component, conventional relay protection, it's hard for conventional relay protection to deal with the problem in surplus power system caused by trend in transfer.With the development of the Smart Grid, higher requirements are needed for relay protection. Therefore, more and more attention are paid to WAP.

WAP was proposed by Bertil Ingelsson ${ }^{[1]}$ from Sweden in 1997, applied to prevent long time voltage collapse of interconnected power grid which realizing stable control of voltage. WAP was applied in relay protection later on and now can be defined as follows: Based on multiple information from power system, the system removes the faulty component fast, reliably and accurately (including both primary protection and back-up protection). The affects for safety and stable operation of power system will be analyzed after removing the fault component and act will be taken to realize relay protection and automatic control simultaneously.

\section{The WACDP Algorithm}

WAP Algorithm. The current research on the WAP system mainly focus on two parts:stability control and relay protection field respectively.The division of the two fields in fact reflects the different understanding of WAP system functions.In reference [1], there are three kinds of function in the power system WAP (stability control) and its corresponding monitoring measurement system: Assuring the safety and stable operation of power grid; acquiring the real-time information of power grid and fully use the transmission capacity of power grid, making it powerful tool for power market operation; providing a more accurate plan for power grid. The wide-area relay protection is using wide-area information to protect the power system. That is,making full use of the all kinds of information collected, enriching the protection function and improving protection performance by applying various protection principle ${ }^{[2]}$.

At present,the study of wide-area relay protection field is mainly concentrated in three aspects: Structure, algorithm, communication system. Research in algorithm mainly includes wide-area relay protection system based on the principle of longitudinal direction, on the distance element and on the principle of current differential protection.

In WAP based on the principle of longitudinal direction, fault diagnosis is realized by direction information from wide-area fault. To increase the output reliability of fault direction element, a 
variety of algorithms can be used to judge the fault direction in each Intelligent Electronic Device (IED).In WAP based on distance element, fault is judged comprehensively by all the action information and switch information from IED in relevant area. As for WAP based on the principle of current differential protection, Kirchhoff Current Law (KCL) is applied, which is the same as the conventional current differential principle. The difference is the protection object. The object is a single electrical component for conventional current protection, while a region, including multiple electrical components, for WACDP.

WACDP Algorithms. The current differential protection principle based on KCL is simple and reliable, with high sensitivity. It has been widely and well used as the primary protection of transmission line, busbar and electrical equipment.

In our work, it has already been learnt about the WACDP algorithms and control strategies: (1) WAP system based on grid calculation method; (2) Zone division of expert system by WACDP; (3) WAMS based power flow transfer identification algorithm; (4) WACDP system based on multi agent; (5) protection algorithm based on tree topology ${ }^{[12]}$; (6) WACDP based on the graph theory method. Each algorithm has its own pros and cons. It is through the acquisition and communication of power network information to realize the WAP.

The Principle of Current Differential Protection. Principle of current differential protection is simple and reliable, and its phase current action criterion is shown in the formula (1):

$$
\begin{aligned}
& \left|\dot{I}_{m}+\dot{I}_{n}\right|>I_{\text {act }} \\
& \left|\dot{I}_{m}+\dot{I}_{n}\right|>k_{\text {res }}\left|\dot{I}_{m}-\dot{I}_{n}\right|
\end{aligned}
$$

Where $\dot{I}_{m}, \dot{I}_{n}$ are the current of both end of the line, $I_{a c t}$ is the threshold of action current value, $k_{\text {res }}$ is the brake coefficient. When external short circuit happens $\dot{I}_{m}=-\dot{I}_{n}$, the action current is 0 . When internal short circuit happens, $\dot{I}_{m}$ and $\dot{I}_{n}$ are in the same phase ,action current is much larger than the braking current. It appears that the criteria above for internal and external fault identification has absolute reliability.

WACDP Based on Graph Theory. There are two key problems in WAP: One is to determine the protection range of every IED, and the other is to divide the related fields of each IED according to the protection scope, that is from which IED the information should be changed when fault happens.

In reference [7] the method of determining the range of IED protection and the determination of its related fields, by using the graph theory method. First, the area of WACDP is divided via topological tree, and the intelligent protection terminal IED is viewed as the research object. When a fault occurs, compare the current in turn and region by region starting with the minimum protection range. If the criterion fits, the differential current method will be stopped and the fault location will be found. Otherwise it will continue to compare, until all the IEDs in the information field are compared by the differential current. If the criterion still does not fit, it can be judged that the area has no fault.

The pros of graph theory are as follows. The protection scope and the related fields can be found by only storing the subgraph into the central processing unit (CPU). When the topology of the network changes, only needed to modify the subgraph and there is no need to modifying the program, which makes the method practical and can be easily enhanced.

\section{WACDP Based on DSP}

DSP is a microprocessor especially designed for processing digital signals. Its main application is real-time and fast implementation of various digital signal processing algorithms. Our work utilizes DSP TMS320F28335 from TI Company, with high accuracy, low cost, low power consumption, high performance, high level of peripheral integration, large data and program memory and more accurate and faster A/D conversion.

System Hardware Block Diagram. The system structure diagram is shown in Fig. 1. Employing 
DSP as the core controller. Data for current differential protection is acquired and converted from current value by $\mathrm{AD}$ converter integrated on the chip. Trip signals and alarm signals are sent from GPIO ports. When a fault occurs and the fault location is determined, GPIO ports will issue trip command to trip circuit breaker, sending out the alarm signal simultaneously.

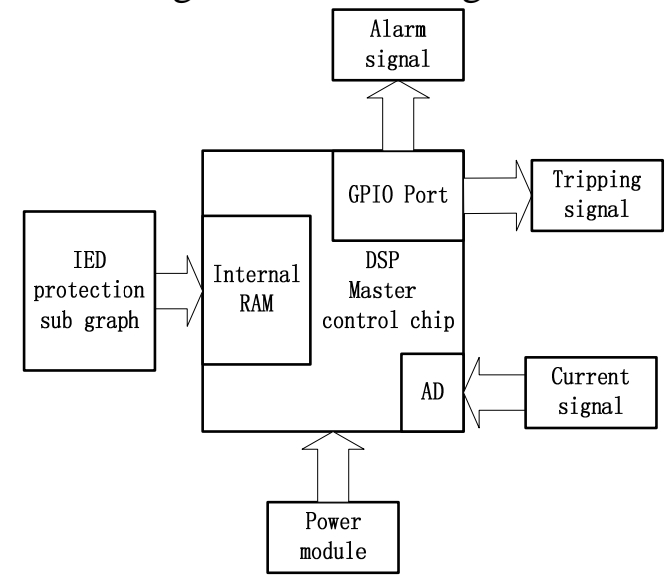

Fig. 1. System structure diagram

AD Sampling. AD sampling module is the DSP internal 12-bit AD converter. First, the current value in the power line is measured and attenuated with a certain proportion in the model. Next, the current signal will be converted to voltage signal using current / voltage (I/V) conversion. And finally after conditioning circuit, the signal will be acceptable for DSP to directly acquire the data.

In order to ensure data accuracy, in this work, 48 points were sampled inside each sine wave period. After digital filteringand waveform restoring, and the current value was obtained via Fourier series algorithm .

Software Flow Chart. As shown in Fig. 2, after system initialization, adjacency matrix reachability matrix and complete incidence matrix are generated according to sub graph. Then, current value is taken via AD conversion module and checked iteratively to detect whether there exists a fault element. If the fault element starts, it will search in the related field to find the fault point, issue the trip command to trip the circuit breaker, and issue a warning signal to alarm staff fault occurs.

\section{Hardware in Loop Experiment}

The RT-LAB real-time simulation platform can directly realize real-time simulation in control, testing and other related fields of the dynamic system model built up by MATLAB/Simulink. Users can achieve the full set of solutions for the project design, real-time simulation, rapid prototyping and hardware loop test on just one platform.

In this work, MATLAB/Simulink is used to build simulation model and simulate the power grid fault. Utilizing both RT-LAB simulation platform and the actual controller DSP. The analog current signal is output from the model's protective installation point. DSP is used sample data and calculate current value, determine whether and where the fault occurs, send out appropriate control command.

The relation among the Simulink model, the RT-LAB simulation platform and the DSP controller is shown in Fig. 3.

Simulation Model. As is shown, Fig. 4 is the simulation system, line voltage is $220 \mathrm{kV}$, CB1 CB12 are circuit breakers, B1 B7 are bus nodes, I1 and I2 are the current of the lines. Line lengths as shown in the Fig.4. Three-phase grounding faults are set at both A and B, respectively.

Protection Simulation. As shown in the system diagram, three-phase short-circuit occurs at the point A. After the first round of the fault, the fault is located between B3 and B4, and a trip signal is issued to trip CB5 and CB6. 


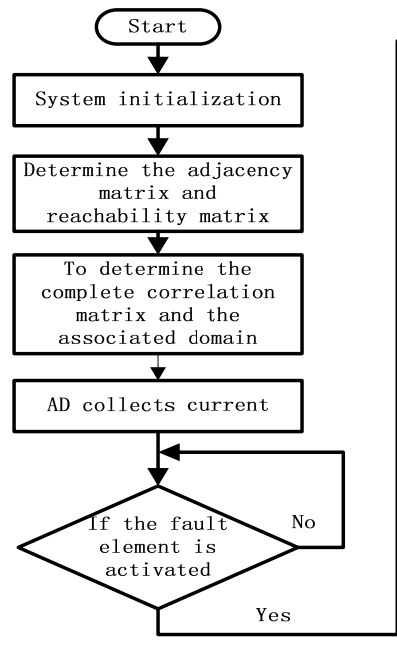

Fig.2. Software flow chart

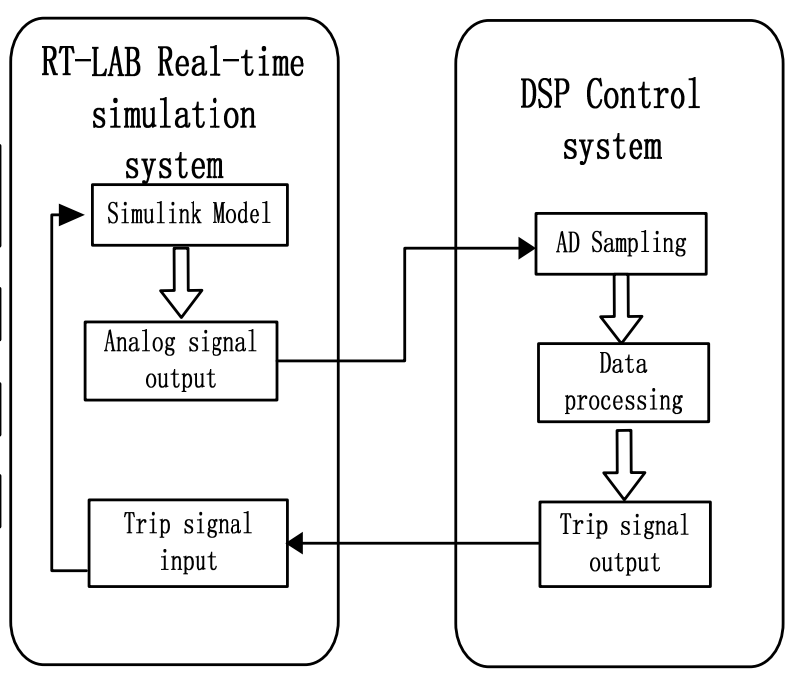

Fig .3. Relation between DSP and RT-LAB

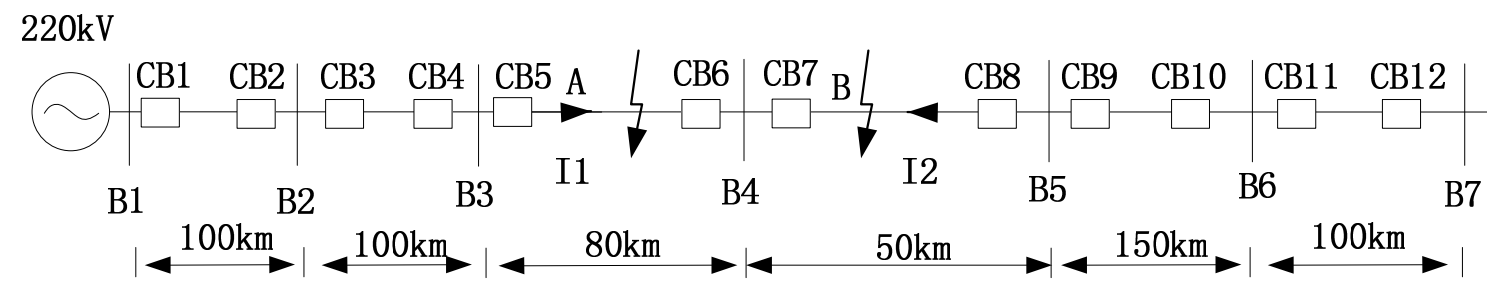

Fig. 4. Simulation system model

In this article, the current mutation method is used. After a fault is detected, recapture the waveform for one period to determine the fault and then sends out trip signal. Waveforms are shown in Fig.5, point A is short circuit point, the tripping signal is sent, the circuit breaker CB5 and CB6 will trip, and the current I1 does down to 0 . Current I2 in the short circuit that has a shock mutation. When the fault line is cut off, the current recovers to stable value, slightly larger than the normal value, but still inside the system capacity range.

When the three-phase short-circuit happens at point $\mathrm{B}$, the situation is similar to point $\mathrm{A}$. After fault detection, the fault point is located between B4 and B5, the circuit breakers CB7 and CB8 will trip. I2 shuts down totally and I1 get larger with transient impact. After shutting down the fault line, I1 returns to normal capacity. Waveforms are shown in Fig.6.

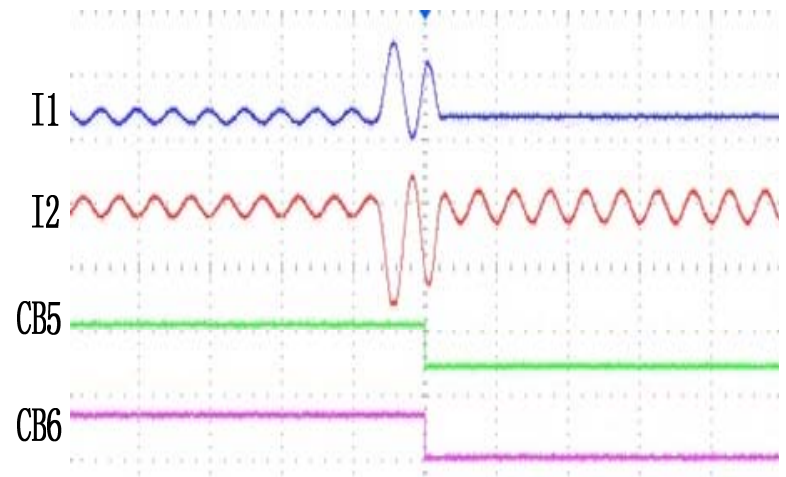

Fig.5. Waveforms for fault point A

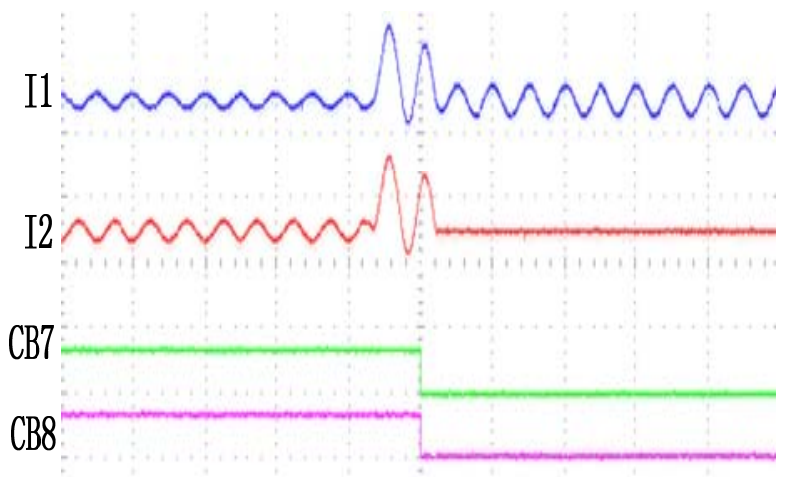

Fig.6. Waveforms for fault point B

\section{Conclusions}

This article introduces the development of WAP and the principle of WACDP. The feasibility of WACDP based on graph theory is verified via DSP28335 controller and RT-LAB simulation platform. 


\section{Acknowledgements}

This work was financially supported by the National Natural Science Foundation of China (51177074, 51407095) and Jiangsu province natural science foundation (BK20151548).

\section{References}

[1] Yunqing Cai, Wang Lei, KipMorison, PrabhaKundur, Zhou Fengquan, Guo Zhizhong. Power Grid Technology, 2004, 08: 20-25. In Chinese

[2] Bao Zhang. Chinese Journal of Electrical Engineering, 2004,07: 5-10. In Chinese

[3] Huifang Wang, Wei Ding, Mengxiao Chen, Wei Xu. Power System Protection and Control, 2012, 01: 145-155. In Chinese

[4] Y. Serizawa, M. Myoujin, K. Kitamura et al. IEEE Transactions on Power Delivery, 1998, 13 (4): 1046-1052.

[5] MIROSLAV BEGOVIC, DANIEL KARLSSON, GARY MICHEL et al. Proceedings of the IEEE, 2005, 93(5) : 876-891.

[6] Lv Y, You D, Wang K, et al. Study on wide-area backup protection system for the smart grid[C]. Electric Utility Deregulation and Restructuring and Power Technologies (DRPT), 2011 4th International Conference on IEEE, 2011: 218 - 224.

[7] Chaohao Cai. Electric Power Science and Engineering, 2009, 09: 7-11. In Chinese

[8] Ingelsson B., Lindstrom P.-O. IEEE Computer Applications in Power, 1997, 10(4) : 30-35.

[9] Changhong Yang . Research on wide area backup protection of transmission line [D]. Southwest Jiao Tong University, 2008. In Chinese

[10] Jun Tang . Research on the wide area current differential protection algorithm of power network [D]. Southwest Jiao Tong University, 2007. In Chinese

[11] Wei Cong, ZhenCun Pan, Jianguo Zhao. Power Grid Technology, 2006, 30 (5): 91-95, In Chinese 